Research review paper

\title{
Fatty acid from the renewable sources: A promising feedstock for the production of biofuels and biobased chemicals
}

\author{
Hui Liu*, Tao Cheng, Mo Xian, Yujin Cao, Fang Fang, Huibin Zou* \\ CAS Key Laboratory of Bio-based Materials, Qingdao Institute of Bioenergy and Bioprocess Technology, Chinese Academy of Sciences, Qingdao 266101, China
}

\section{A R T I C L E I N F O}

\section{Article history:}

Received 16 July 2013

Received in revised form 11 December 2013

Accepted 13 December 2013

Available online 19 December 2013

\section{Keywords:}

Fatty acid

Biofuels

Biobased chemicals

Multiscale analysis

Interdisciplinary study

Renewable biomass

\begin{abstract}
A B S T R A C T
With the depletion of the nonrenewable petrochemical resources and the increasing concerns of environmental pollution globally, biofuels and biobased chemicals produced from the renewable resources appear to be of great strategic significance. The present review described the progress in the biosynthesis of fatty acid and its derivatives from renewable biomass and emphasized the importance of fatty acid serving as the platform chemical and feedstock for a variety of chemicals. Due to the low efficient conversions of lignocellulosic biomass or carbon dioxide to fatty acid, we also put forward that rational strategies for the production of fatty acid and its derivatives should further derive from the consideration of whole bioprocess (pretreatment, saccharification, fermentation, separation), multiscale analysis and interdisciplinary combinations (omics, kinetics, metabolic engineering, synthetic biology, fermentation and so on).
\end{abstract}

(c) 2013 Elsevier Inc. All rights reserved.

\section{Contents}

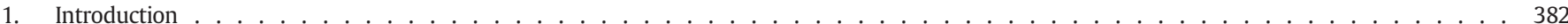

2. Major routes for the microbial production of fatty acid and its derivatives. . . . . . . . . . . . . . . . . . . . . . . . . . . . . 384

2.1. Conversion of lignocellulosic biomass to fatty acid and its derivatives . . . . . . . . . . . . . . . . . . . . . . . 384

2.2. Conversion of solar energy and carbon dioxide into fatty acid and its derivatives in photosynthetic algae . . . . . . . . . . . . . . . 384

3. Metabolic engineering for fatty acid production . . . . . . . . . . . . . . . . . . . . . . . . . . . . . 384

4. Metabolic engineering for the fatty acid derivatives. . . . . . . . . . . . . . . . . . . . . . . . . . . . . . 385

4.1. The fuels and chemical products in the downstream pathways next to fatty acid biosynthesis pathway . . . . . . . . . . . . . . . 385

4.2. The fuels and chemical products deriving from fatty acid through the key intermediate metabolite AcCoA . . . . . . . . . . . . . . . . . 386

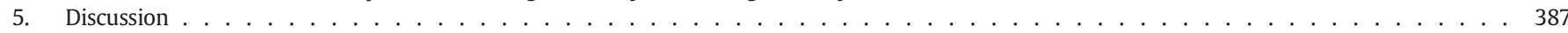

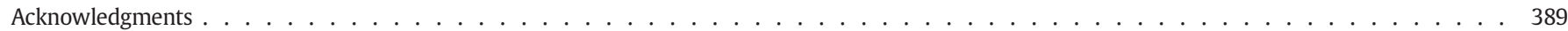

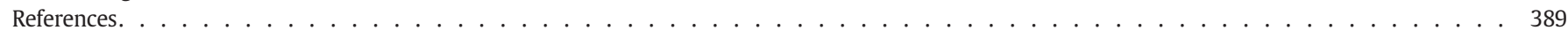

\section{Introduction}

The traditional oleochemical industry has processed vegetable oils and animal fats for more than 100 years. With the depletion of the nonrenewable petrochemical resources, around $20 \%$ of 150 million tonnes of fats and oils have to be transformed into biofuels and chemical products annually in recent years (Schörken and Kempers, 2009). The increased demand for the fuels and chemicals produced from the fatty

\footnotetext{
* Corresponding authors. Tel.: + 86532 80662766; fax: + 8653280662765.

E-mail addresses: liuhui@qibebt.ac.cn (H. Liu),zouhb@qibebt.ac.cn (H. Zou).
}

acid of plant and animal oils resulted in competition with food, higher prices, questionable land-use practices and environmental concerns associated with oil production (Hill et al., 2006; Steen et al., 2010). In order to cope with the increasing energy costs and environmental concerns, sustainable renewable fuels and chemicals in Fig. 1 should be manufactured from free fatty acid directly through microbial conversions in our opinion. In a meanwhile, the free fatty acid should be derived from various types of biomass such as lignocellulosic biomass, nonedible alga oils or waste plant/animal oils and so on.

In the present review, we demonstrated that free fatty acid from renewable resources would be a promising potential feedstock for fuels and chemical manufacture in the future. Due to the development of 


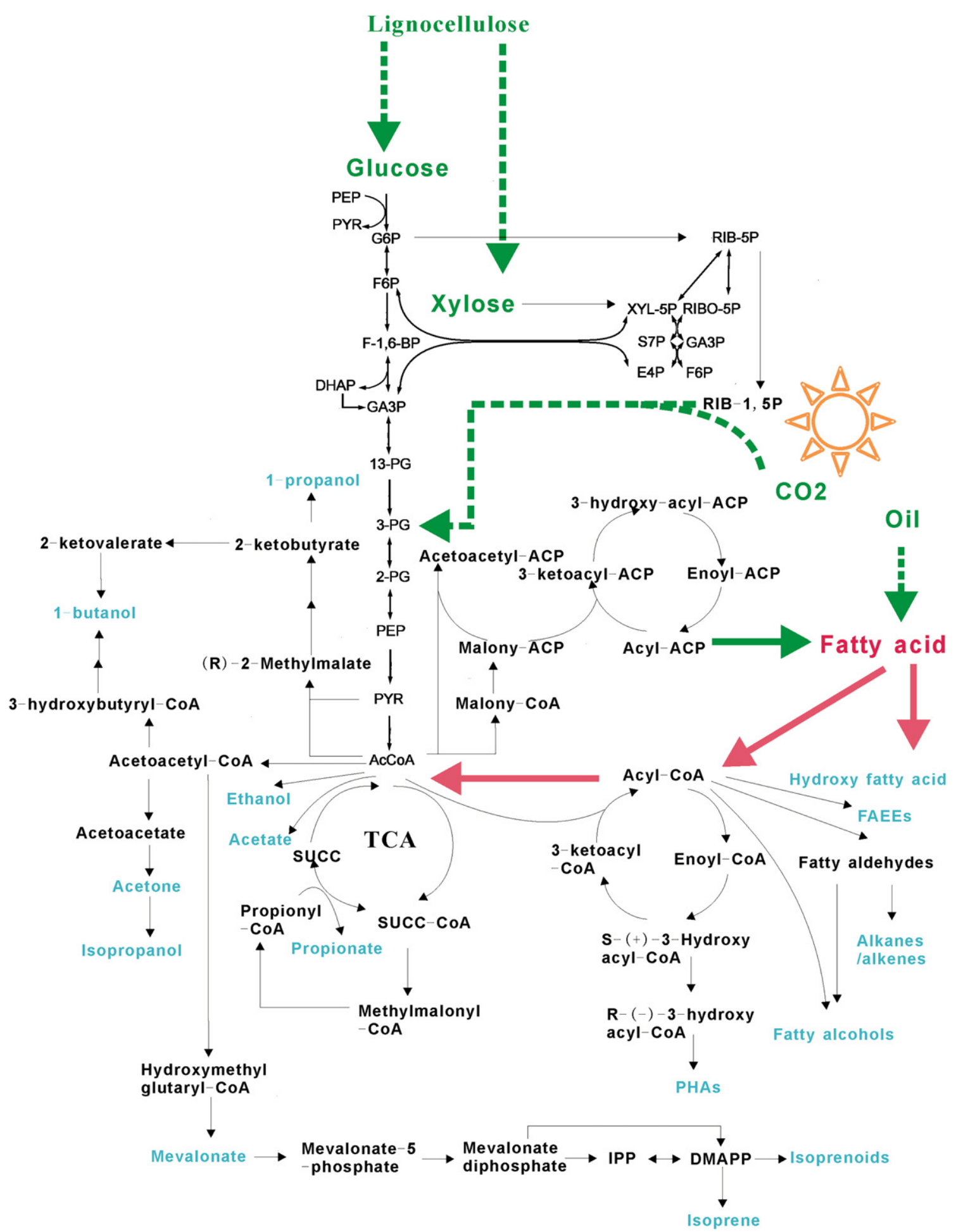

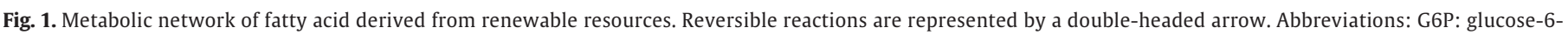

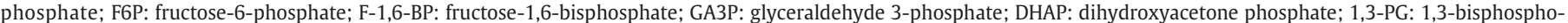

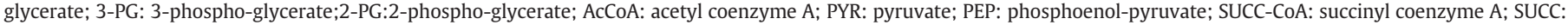

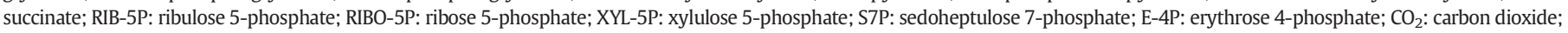
IPP: isopentenylpyrophosphate; DMAPP: dimethylallyl pyrophosphate.

metabolic engineering, the free fatty acid can presently be obtained from the renewable resources like lignocellulosic sugars and carbon dioxide through microbial conversion (Liu et al., 2011; Steen et al., 2010), which has the following advantages: renewability, short production cycle, less labor requirement, less affection by venue, season and climate, and easier to scale up (Li et al., 2008). Furthermore, as the direct precursor of advanced biofuels, the cost-effective production process of free fatty acid is greatly essential for the production of alkanes, biodiesel, fatty alcohols and so on. Finally, free fatty acid could also be an important feedstock for the biosynthesis of platform chemicals 
such as C4 and C5 biobased products through microbial conversion (Dellomonaco et al., 2010). This is because free fatty acid can be efficiently metabolized to the key intermediate metabolite acetyl coenzyme A with the carbon recovery of $100 \%$. Then the acetyl-CoA can be converted to biofuels (ethanol, butanol) and chemicals (acetate, mevalonate, isoprenoids, PHAs).

This review also described the major routes for the production of fatty acid and its derivatives (especially the consolidated bioprocessing) from renewable resources. Although substantial achievements have been made in recent years, the production of fatty acid and its derivatives from renewable resources is still in proof-in-concept stages. The efficient conversions of lignocellulosic biomass or carbon dioxide to fatty acid remain a great challenge to be solved. Therefore, we further discussed the rational strategies for the improvement of fatty acid and its derivative production from renewable resources.

\section{Major routes for the microbial production of fatty acid and its derivatives}

The renewable sources for the production of fatty acid and its derivatives are primarily composed of lignocellulosic biomass, carbon dioxide, the nonedible alga oils, waste plant/animal oils and so on. Recent advances in the conversion processes of renewable resources offer the opportunities for the cost-effective production of fatty acid and its derivatives.

\subsection{Conversion of lignocellulosic biomass to fatty acid and its derivatives}

Many lignocellulosic biomass feedstocks can be used for the production of fatty acid and its derivatives, which include agricultural residues, wood residues, and waste streams (Aristidou and Penttila, 2000). The technologies for lignocellulose conversion usually commence with a physical and/or chemical pretreatment, which could disrupt the complex structure and render the polymeric sugar more accessible to subsequent biology conversion process including consolidated bioprocessing (CBP). The biology conversion process typically involves enzymatic methods of saccharification and fermentation of hexose sugars and pentose sugars. You et al. demonstrated one-pot enzymatic conversion of pretreated biomass to starch through nonnatural enzymatic pathway (You et al., 2013), which was an important novel method for the conversion of cellulose. The starch and the remaining cellulose can both be further hydrolyzed to glucose suitable for fatty acid and its derivative production. Heretofore, the costs of both pretreatment and enzymes, and low hydrolysis rate of cellulose and hemicellulose to sugars and the low-efficient utilization of hemicellulose hydrolysis (pentoses and hexoses) are still main drawbacks constraining the industrial production process of fatty acid and other biobased products from lignocellulosic biomass (Grange et al., 2010; Ragauskas et al., 2006; Sims et al., 2010).

For the cost-effective fatty acid production from lignocellulosic biomass, the fatty acid producer should be engineered to express a heterologous cellulase/hemicellulase system to enable cellulose and hemicellulose utilization. Previous reports suggested that Escherichia coli could be engineered to produce fatty acid and fatty acid-derivatives from glucose (Liu et al., 2012; Lu et al., 2008; Steen et al., 2010). While fortunately E. coli could utilize both pentoses and hexoses, Steen et al. expressed the hemicellulases Xynlol3 and Xsa in E. coli strains, which resulted in the fatty acid ethyl ester production directly from birchwood xylan (Steen et al., 2010). This was the first successful consolidated bioprocessing (CBP) combining enzyme production, hemicellulose hydrolysis and fermentation together in E. coli for fatty acid derivatives production. Later, Bokinsky et al. synthesized three advance biofuels (FAEE, butanol and pinene) from ionic liquid pretreated switchgrass using engineered $E$. coli, which represented a major advance toward realizing CBP (Bokinsky et al., 2011). However, all these efforts are in proof-of-concept stages. The relevant advances were far from enough and the great potential of CBP has not been realized using microorganisms available today (Wen et al., 2010).

\subsection{Conversion of solar energy and carbon dioxide into fatty acid and its derivatives in photosynthetic algae}

Conversion of solar energy and carbon dioxide into fatty acid in photosynthetic algae was a promising strategy to develop sustainable biofuels and chemicals. Main advantages of photosynthetic algae system are as follows: (1) high photo conversion efficiency, (2) a reliable and continuous supply all-year-round, (3) utilization of salt and waste water streams, and (4) coupling $\mathrm{CO}_{2}$-neutral fuel production with sequestration (Schenk et al., 2008). The reports on the production of fatty acid and its derivatives by Cyanobacteria have raised great attentions in recent years. Liu et.al described that manipulation of fatty acid metabolic pathway and deletion of S layer protein led to overproduction and secretion of fatty acid in Synechocystis sp. PCC6803 (Liu et al., 2011). Lu et al. reviewed the photosynthetic production of fatty acid-based biofuels in genetically engineered Cyanobacteria (Lu, 2010). Fatty acyl-CoA synthase gene knock-out contributed to the production of free fatty acid with the yields of $6.4 \mathrm{nmol} / \mathrm{ml} / \mathrm{OD}_{750}$ for Synechocystis $\mathrm{sp}$. PCC6803 greatly and $8.4 \mathrm{nmol} / \mathrm{ml} / \mathrm{OD}_{750}$ for PCC7942 (Kaczmarzyk and Fulda, 2010; Lu, 2010). Alkane biosynthesis exists in numerous organisms, while the new pathway for alkane biosynthesis in Cyanobacteria was just recently discovered by Schirmer et al. The pathway consists of an acyl-acyl carrier protein reductase and an aldehyde decarbonylase, which together convert intermediates of fatty acid metabolism to alkanes and alkenes. Heterologous expression of alkane operon in E. coli leads to the production and secretion of $\mathrm{C} 13$ to $\mathrm{C} 17$ mixtures of alkanes and alkenes (Schirmer et al., 2010). Wang et al. engineered Cyanobacteria to improve photosynthetic production of alkanes through redirecting the carbon flux to acyl-ACP and overexpression of alkane biosynthetic genes (Wang et al., 2013). Zhang et al. identified that the ferredoxin/ ferredoxin-NADP ${ }^{+}$reductase electron transfer system could support aldehyde-deforming oxygenase activity in Synechococcus elongatus PCC7942 (Zhang et al., 2013).

\section{Metabolic engineering for fatty acid production}

There have been many reports on the development of fatty acid producer recently (Table 1) (Liu et al., 2012). The Cyanobacteria was modified to produce and secrete fatty acids to avoid costly biomass recovery in photosynthetic microbial biofuel production. The fatty acid titer was increased to $197 \pm 14 \mathrm{mg} / \mathrm{L}$ by adding codon-optimized thioesterase genes and weakening polar cell wall layers (Liu et al., 2011). Besides the oleaginous microorganisms like microalgae, fungi and yeast, the industrial strain $E$. coli is becoming a new focus for fatty acid production and some breakthroughs have been made (Steen et al., 2010). The formation of malonyl-CoA from acetyl-CoA catalyzed by acetyl-CoA carboxylase (ACC) is the first key rate-limiting step of fatty acid biosynthetic pathway (Davis et al., 2000; Li and Cronan, 1993). The overexpression of ACC in E. coli has led to an increase in the rate of fatty acid synthesis (Davis et al., 2000). Expression of bacterial or plant acyl-ACP thioesterases can reduce the cellular acyl-ACP concentration, decrease the feedback inhibition of fatty acyl-ACP and increase fatty acid production in E. coli (Cho and Cronan, 1995; Davis et al., 2000; Jiang and Cronan, 1994; Voelker and Davies, 1994). A 7-fold increase of total fatty acid production was observed by eliminating $\beta$-oxidation in E. coli, overexpressing ACC and expressing plant acyl-ACP thioesterases from Umbellularia californica in low copy number plasmids, and the fatty acid was successfully converted to alkane by a catalytic reaction (Lennen et al., 2010). In the fadD deleted strain, coexpression of three genes, thioesterases from Cinnamomum camphorum and E. coli, as well as ACC from E. coli resulted in 20 -fold enhancement of total fatty acid production in shake flasks. $2.5 \mathrm{~g} / \mathrm{L}$ fatty acids were produced by this engineered strain in fed-batch cultivation, but no cultivation 


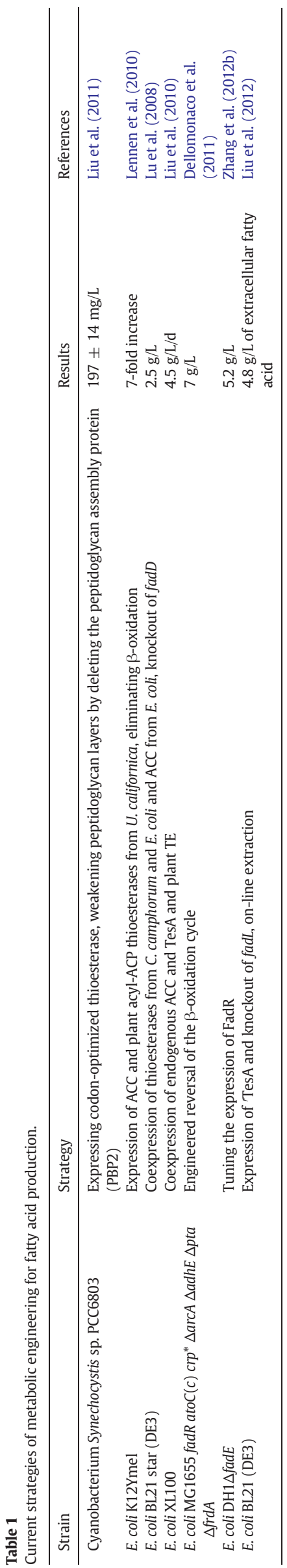

optimization was further conducted (Lu et al., 2008). With further efforts done to increase fatty acid production by targeting the genes in the fatty acid biosynthetic pathway, the productivity was increased to $4.5 \mathrm{~g} / \mathrm{L} / \mathrm{d}$ (Liu et al., 2010). The reversal of the $\beta$-oxidation cycle was also engineered as a metabolic platform to synthesize fatty acid in E. coli with various chain lengths above 10 carbons at high titer of $7 \mathrm{~g} / \mathrm{L}$ and yield of $0.28 \mathrm{~g} / \mathrm{g}$ glucose (Dellomonaco et al., 2011).

Generally speaking, the construction of genetically engineered strains for fatty acid production mainly focused on the terminal pathway, such as the overexpression of the rate-limiting enzyme and removing feedback inhibitions in the fatty acid biosynthetic pathway. However, these approaches did not always result in great increment because the kinetic fermentative behavior of strains and the integrated function of the global regulation and metabolic network were neglected (Lee et al., 2009; Liao et al., 1996; Liu et al., 2012; Stephanopoulos, 2007). Firstly, the strategy of metabolic engineering for fatty acid production should be the cyclic optimization between cultivation performance and strain improvements (Lee et al., 2009). Using developed strains, cultivation process should be performed. On the basis of cultivation performance, further metabolic engineering should be carried out for the strain improvement. Secondly, the global regulation and metabolic networks should also be paid attention to. Zhang et al. found that FadR could enhance fatty acid production by globally tuning the expression levels of many genes to optimal levels. By tuning the expression of FadR in E. coli, the fatty acid titer was increased by 7.5 -fold to $5.2 \pm 0.5 \mathrm{~g} / \mathrm{L}$, with the yield accounting for $73 \%$ of the theoretical yield (Zhang et al., 2012b).

\section{Metabolic engineering for the fatty acid derivatives}

4.1. The fuels and chemical products in the downstream pathways next to fatty acid biosynthesis pathway

$\omega$-Hydroxy fatty acids are excellent monomers for synthesizing a unique family of polyethylene-like biobased plastics. Wang et al. reported the biosynthesis of long chain hydroxy fatty acid directly from glucose without the need of exogenous fatty acid (Table 2). High specificity production of $\mathrm{C} 12$ and C14 HFAs was achieved by expressing thioesterases BTE and 'TesA and fatty acid hydroxylase P450 E. coli. By further knocking out the endogenous fadD gene, $117.0 \mathrm{mg} / \mathrm{L}$ HFAs was finally obtained in shake flasks (Wang et al., 2012). Lu et al. reported that 16 genes encoding 6 cytochrome P450s, 4 fatty alcohol oxidase, and 6 alcohol dehydrogenases from the Candida tropicalis were eliminated to construct strains capable of converting a range of fatty acids to the corresponding $\omega$-hydroxy fatty acids. A strain that reintegrated a select $\mathrm{P} 450$ resulted in the biotransformation of methyl tetradecanoate to 14-hydroxytetradecanoic acid with high yield of $87 \%$ (Table 2). A total of $174 \mathrm{~g} / \mathrm{L}$ of 14 -hydroxytetradecanoic acid and $6.1 \mathrm{~g} / \mathrm{L}$ of 1,14-tetradecanedioic acid were obtained with the addition of $200 \mathrm{~g} / \mathrm{L}$ methyl tetradecanoate after $148 \mathrm{~h}$ of biotransformation ( $\mathrm{Lu}$ et al., 2010).

Biodiesel is composed of fatty acid methyl and ethyl esters (FAMEs and FAEEs), which is usually produced by transesterification of plant and animal oils. The microbial production of FAEEs (Table 2) was achieved by heterologous expression in E. coli of Zymomonas mobilis pyruvate decarboxylase (PDC) and alcohol dehydrogenase (AdhB) and the unspecific acyltransferase from Acinetobacter baylyi strain ADP1. $1.28 \mathrm{~g} / \mathrm{L}$ of FAEEs and $26 \%$ of FAEE content in dry cell weight were achieved in the presence of glucose and oleic acid (Kalscheuer et al., 2006). To obviate the need to feed external fatty acid, $400 \mathrm{mg} / \mathrm{L}$ of FAEEs was produced from glucose and ethanol by E. coli through expressing a wax-ester synthase (AtfA) and FadD and 'TesA. Furthermore, to obviate the need to feed ethanol, FAEEs derived from endogenously produced fatty acids and ethanol were produced in E. coli by directly expressing the $Z$. mobilis genes pdc and $a d h B$ (Steen et al., 2010). Besides Steen and Kalscheuer et al.'s works on FAEE production, further 


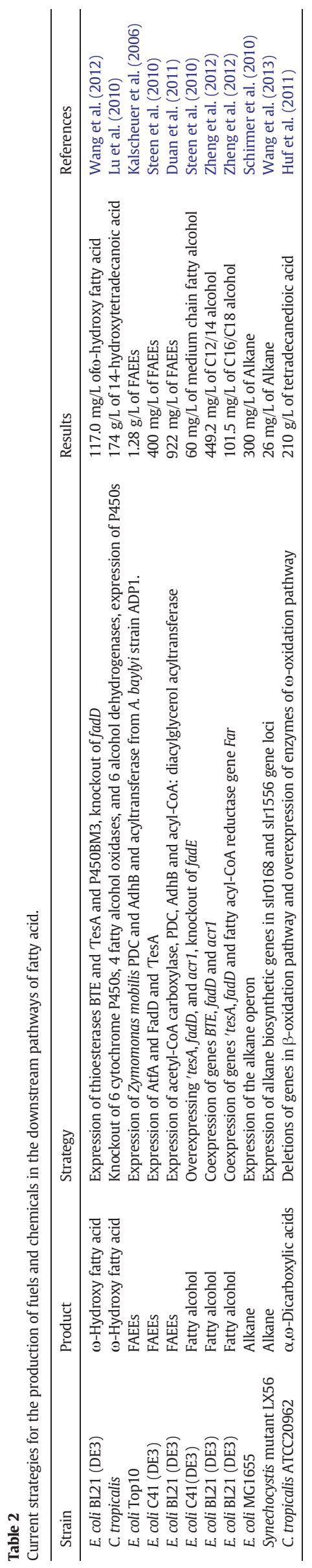

improvements were made by Duan et al. Overexpression of acetyl-CoA carboxylase in E. coli was employed to increase the pool of fatty acyl$\mathrm{CoA}$ and up to FAEE titer of $922 \mathrm{mg} / \mathrm{L}$ was finally reached under optimized fermentation conditions (Duan et al., 2011).

Fatty alcohols and wax esters are widely used in soaps, detergents, cosmetic additives, pheromones and flavoring compounds, and potentially as biofuels. The biosynthesis of fatty alcohols mainly employs the de novo fatty acid synthetic pathway, which are sequentially catalyzed by thioesterase, acyl-Coenzyme A synthase and fatty acyl-CoA reductase (Table 2). Overexpressing 'tesA, fadD, and acr1 in E. coli C41(DE3) $\Delta$ fadE resulted in the production of medium chain fatty alcohols up to $60 \mathrm{mg} / \mathrm{L}$ (Steen et al., 2010). Zheng et.al firstly reported the selective production of C12/14 and C16/18 alcohols. By coexpression of genes BTE, fadD and acr1, $449.2 \mathrm{mg} / \mathrm{L} \mathrm{C12/14}$ alcohol was obtained, with C12/14 alcohol accounting for $75 \%$ of the total fatty alcohol production. By coexpression of genes 'tesA, fadD and fatty acyl-CoA reductase gene Far, $101.5 \mathrm{mg} / \mathrm{LC16}$ C18 alcohol was obtained, with C16/C18 alcohol accounting for $89.2 \%$ of the total fatty alcohol production (Zheng et al., 2012).

The engineering of $E$. coli to produce waxes through the fatty alcohol pathway (FAR) directly from simple sugars was demonstrated by Steen et al. (2010). In the similar fashion as biodiesel, an E. coli strain which was engineered to express 'tes $A$, fadD, mFar1 and atfA could produce wax esters including tetradecanoate hexadecyl ester, hexadecanoate hexadecyl ester, 9-hexadecenoate hexadecyl ester and 9-octadecenoate octadecyl ester from glucose as the sole carbon source.

Alkanes, the major constituents of gasoline, diesel and jet fuel, are biosynthesized from intermediates of fatty acid metabolism. An alkane biosynthesis pathway from Cyanobacteria consisting of an acyl-acyl carrier protein reductase and an aldehyde decarbonylase was discovered. The heterologous expression of the alkane operon in E. coli leads to the production and secretion of $\mathrm{C} 13$ to $\mathrm{C} 17$ mixtures of alkanes and alkenes (Table 2). Alkane titers were over $300 \mathrm{mg} / \mathrm{L}$ and more than $80 \%$ of hydrocarbons were found outside the cells (Schirmer et al., 2010). Furthermore, the alkane titer of $26 \mathrm{mg} / \mathrm{L}$ was produced by Synechocystis mutant LX56 harboring alkane biosynthetic genes overexpressed in slr0168 and slr1556 gene loci (Wang et al., 2013).

$\alpha, \omega$-Dicarboxylic acids are versatile chemical intermediates of different chain lengths and are used as raw material for the preparation of perfumes, polymers, adhesives and macrolide antibiotics. The strategies for industrial production of long chain dicarboxylic acids via metabolic engineering and bioprocess engineering are described (Huf et al., 2011). The preparation of high level DCA-producing strain mainly focused on the deletions of genes in $\beta$-oxidation pathway and overexpression of enzymes of $\omega$-oxidation pathway (Table 2 ). Production of tetradecanedioic acid (C14) up to $210 \mathrm{~g} / \mathrm{L}$ was achieved with a DCA production rate of $1.3 \mathrm{~g} / \mathrm{L} / \mathrm{h}$ (Huf et al., 2011; Picataggio et al., 1992).

4.2. The fuels and chemical products deriving from fatty acid through the key intermediate metabolite AcCoA

Fatty acid can be efficiently converted to acetyl coenzyme A through $\beta$-oxidation pathway with the carbon recovery of $100 \%$. The acetyl-CoA is the key intermediate metabolite for many fuels and chemical biosynthesis such as isoprenoids, mevalonate, acetone, succinate, PHAs and so on. Therefore, if the fatty acid can be efficiently manufactured from carbon dioxide by photosynthetic reaction in algae or from lignocellulosic biomass, or from waste plant/animal oils, the fuels and chemical products derived from acetyl-CoA can be produced from the fatty acid feedstock. However, nowadays only a few reports described engineering microorganism for the conversion of fatty acid to chemical products (Table 3).

Polyhydroxyalkanoates (PHAs) are a family of biodegradable polyesters, which accumulated as carbon and energy sources in many microorganisms. Engineering of fatty acid metabolism for the production of PHAs in E. coli was described by Park et.al, which included manipulations of the fatty acid $\beta$-oxidation pathway and fatty acid de novo 
biosynthesis pathways. The inhibition of FadA and FadB in $\beta$-oxidation pathway and the expression of enoyl-CoA hydratase and 3-ketoacylAcp reductase contributed to the overproduction of PHAs from fatty acid. The representative production of PHA by engineered E. coli was $21.5 \mathrm{~g} / \mathrm{L}$ with the productivity of $0.5 \mathrm{~g} / \mathrm{L} / \mathrm{h}$ (Park et al., 2001).

Dellomonaco et.al engineered several native and heterologous fermentative pathways to enable the efficient synthesis of fuels and chemicals from fatty acid in E. coli (Dellomonaco et al., 2010). The production of ethanol at a yield of $0.48 \mathrm{~g} / \mathrm{g}$ C16:0 was achieved by overexpression of AdhE* (an aerotolerant mutant of acetaldehyde/alcohol dehydrogenase) in E. coli strain MG1655. Incomplete oxidation of $\mathrm{C} 16: 0$ resulted in the accumulation of caproic acid (C6:0). The metabolism of caproic acid in E. coli requires expression of the fad regulon and ato operon. The fadR* and atoC(Con) phenotype in MG1655 led to the constitutive expression of fad regulon and ato operon. Overexpression of AdhE* $^{*}$ in the strain MG1655 fadR* atoC(Con) finally resulted in the production of ethanol at a yield of $1.06 \mathrm{~g} / \mathrm{g} \mathrm{C16:0,} \mathrm{which} \mathrm{is} \mathrm{twice}$ the maximum theoretical value from sugars. As in ethanol-producing strains, the overexpression of genes in the butanol biosynthetic pathway from Clostridium acetobutylicum and atoB from E. coli in the strain MG1655 $\triangle a d h E$ fadR* atoC(Con) resulted in butanol yields up to 3-fold higher than those reported previously. Overexpression of the phosphotrans-acetylase (PTA)-acetate kinase (ACK) pathway in MG1655 fadR* atoC(Con) led to $35 \mathrm{~g} / \mathrm{L}$ acetate with a yield of $1.10 \mathrm{~g} / \mathrm{g}$ C16:0, exhibiting yield surpassed those reported for $E$. coli strains utilizing glucose as carbon sources. Similarly, the acetone yield of $0.37 \mathrm{~g} / \mathrm{g}$ C16:0 was achieved by simultaneous overexpression of the acetateproducing ACK-PTA pathway, along with the synthetic acetone pathway from C. acetobutylicum, which surpassed the yields previously reported for engineered E. coli. As in the case of former products, isopropanol, succinic acid and propionic acid production from fatty acid was also investigated in the fadR* atoC(Con) strains with the positive results observed.

\section{Discussion}

Many breakthroughs have been made for the biosynthesis of fatty acid and its derivatives through metabolic engineering in the last decade. Importantly, Gonzalez et al. successfully engineered $E$. coli strains serving as microbial platforms for the conversion of fatty acid-rich feedstocks (Dellomonaco et al., 2010), which suggested that fatty acid could be a promising feedstock and platform chemicals for the production of biofuels and biochemicals. Subsequently, the efficient conversion of lignocellulosic biomass or carbon dioxide to fatty acid would be a major challenge to be solved. We believed that the bottlenecks of this research direction could be identified and solved by the whole consideration of the production process, multiscale analysis and interdisciplinary study. Considering the application of the systems methods in amino acid production, the construction of L-valine producer is a successful example, which reflects the multiscale and interprinciple strategies (Lee et al., 2009). The target genes to be engineered were identified by transcriptome profiling and their desirable expression levels were predicted by in silico flux response analysis. Further improvements were made through transporter and exporter engineering. Finally, the strain was able to produce L-valine with a high yield of $0.378 \mathrm{~g} / \mathrm{g}$ glucose (Park and Lee, 2008).

Rational strategies for the fatty acid and its derivative production should derive from the consideration of whole bioprocess, multiscale analysis and interdisciplinary study in our opinion (Fig. 2). Improving the efficiency and yield of fatty acid and its derivatives further will require more potent and novel technologies, such as more biocompatible pretreatment methods, rational design of reactors, the process integrations, the strategies of strain improvements derived from the systemlevel analysis and so on. In this direction some progress has been made recently for fatty acid and its derivatives production. However, it is still far from enough. Firstly, both strain improvements and 


\section{Challenge}

(1) low efficiency in carbon utilization (C1, C5, C6) from lignocellulose or carbon dioxide;

(2) low fatty acid titer and yield
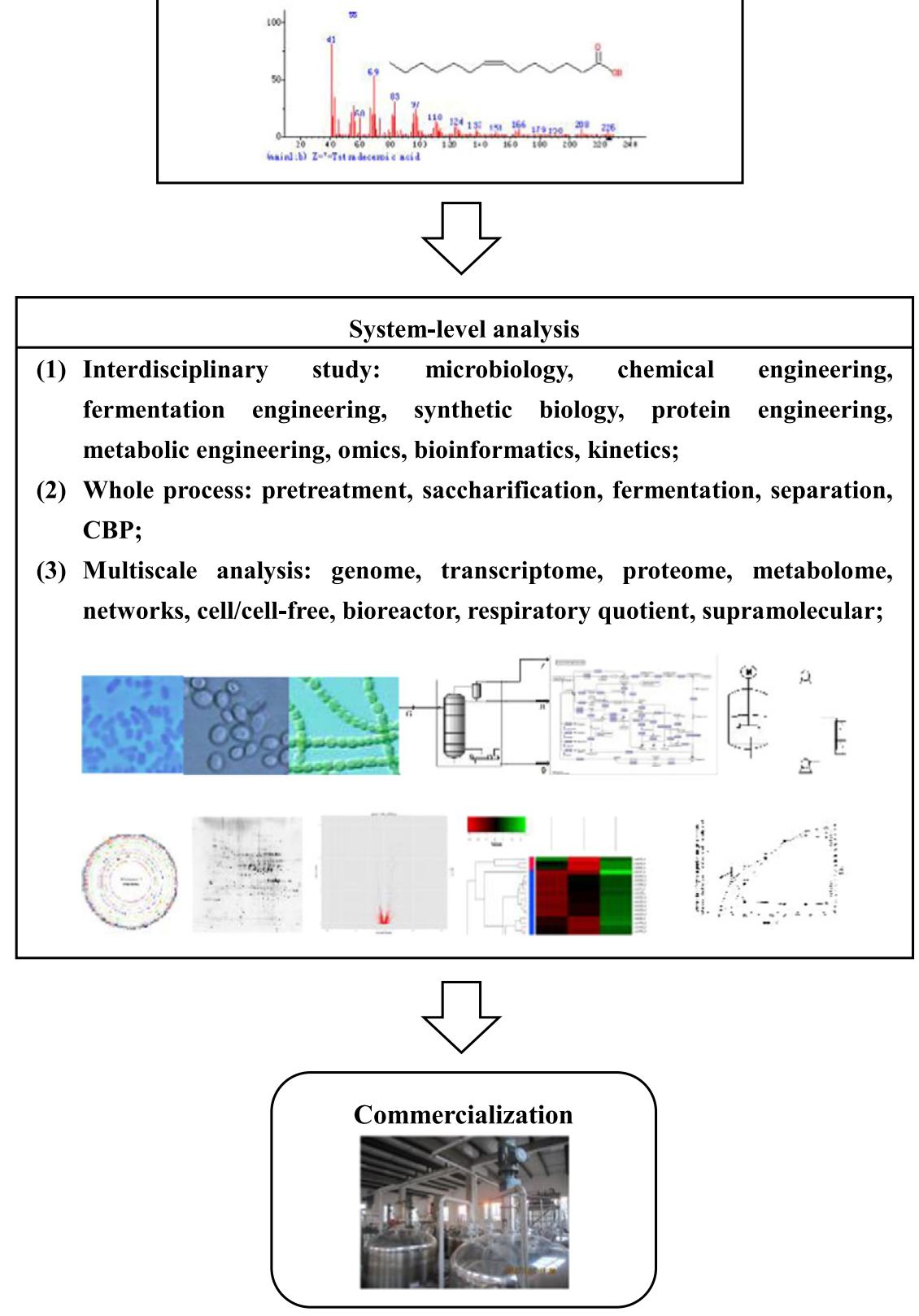

Fig. 2. The strategies for the commercialization of fatty acid and its derivative production from renewable sources.

cultivation process strategies based on the mass transfer kinetics were applied to increase extracellular fatty acid production by engineered E. coli. Under optimal conditions, the E. coli strain (pACY-'tesA- $\Delta$ fadL) produced $4.8 \mathrm{~g} / \mathrm{L}$ extracellular fatty acid and the cyclic optimization between cultivation performance and strain improvements should be further carried out (Liu et al., 2012). Secondly, a combination of biotechnology and heterogeneous catalysis is carried out to produce undecane with a yield of $0.44 \mathrm{~g} / \mathrm{L}$ and an enriched alkane stream that can be recycled for continuous extractions (Lennen et al., 2010). Thirdly, a cell-free system was developed for investigation of fatty acid biosynthesis and its regulation in E. coli. The strong dependence of fatty acid synthesis on malonyl-CoA availability was verified (Liu et al., 2010). The cell-free systems could also be used to identify bottlenecks in fatty acid biosynthesis in other organisms such as algae, plants and yeasts. Fourthly, the genome transcriptional analysis and target proteomics were conducted to study the mechanism of FadR enhancing fatty acid yield, which indicated that FadR could tuning the globally gene expression levels of fatty acid biosynthesis, degradation and membrane transport to optimal levels (Zhang et al., 2012b). Fifthly, Zhang et al. developed a dynamic sensor-regulator system (DSRS), which could regulate the expression of genes involved in the fatty acid-based chemicals production through sensing the concentration of key intermediate acyl-CoA. The DSRS increased the titer of FAEE to $1.5 \mathrm{~g} / \mathrm{L}$ and improved the stability of FAEE-producing strains (Zhang et al., 2012a). 


\section{Acknowledgments}

This work was supported by the National Natural Science Foundation (20872075) and National Key Technology R\&D Program (No. 2012BAD32B06).

\section{References}

Aristidou A, Penttila M. Metabolic engineering applications to renewable resource utilization. Curr Opin Biotechnol 2000;11:187-98.

Bokinsky G, Peralta-Yahya PP, George A, Holmes BM, Steen EJ, Dietrich J, et al. Synthesis of three advanced biofuels from ionic liquid-pretreated switchgrass using engineered Escherichia coli. Proc Natl Acad Sci 2011;108:19949-54.

Cho $\mathrm{H}$, Cronan JE. Defective export of a periplasmic enzyme disrupts regulation of fatty acid synthesis. J Biol Chem 1995;270:4216-9.

Davis MS, Solbiati J, Cronan JE. Overproduction of acetyl-CoA carboxylase activity increases the rate of fatty acid biosynthesis in Escherichia coli. J Biol Chem 2000;275: 28593-8.

Dellomonaco C, Rivera C, Campbell P, Gonzalez R. Engineered respiro-fermentative metabolism for the production of biofuels and biochemicals from fatty acid-rich feedstocks. Appl Environ Microbiol 2010;76:5067-78.

Dellomonaco C, Clomburg JM, Miller EN, Gonzalez R. Engineered reversal of the [bgr]-oxidation cycle for the synthesis of fuels and chemicals. Nature 2011;476: 355-9.

Duan Y, Zhu Z, Cai K, Tan X, Lu X. De novo biosynthesis of biodiesel by Escherichia coli in optimized fed-batch cultivation. PLoS One 2011;6:e20265.

Grange D, Haan R, Zyl W. Engineering cellulolytic ability into bioprocessing organisms. Appl Microbiol Biotechnol 2010;87:1195-208.

Hill J, Nelson E, Tilman D, Polasky S, Tiffany D. Environmental, economic, and energetic costs and benefits of biodiesel and ethanol biofuels. Proc Natl Acad Sci 2006;103: $11206-10$.

Huf S, Krügener S, Hirth T, Rupp S, Zibek S. Biotechnological synthesis of long-chain dicarboxylic acids as building blocks for polymers. Eur J Lipid Sci Technol 2011;113:548-61.

Jiang P, Cronan Jr JE. Inhibition of fatty acid synthesis in Escherichia coli in the absence of phospholipid synthesis and release of inhibition by thioesterase action. J Bacterio 1994;176:2814-21.

Kaczmarzyk D, Fulda M. Fatty acid activation in Cyanobacteria mediated by acyl-acyl carrier protein synthetase enables fatty acid recycling. Plant Physiol 2010;152:1598-610.

Kalscheuer R, Stolting T, Steinbuchel A. Microdiesel: Escherichia coli engineered for fue production. Microbiology 2006;152:2529-36.

Lee SY, Kim HU, Park JH, Park JM, Kim TY. Metabolic engineering of microorganisms: general strategies and drug production. Drug Discov Today 2009;14:78-88.

Lennen RM, Braden DJ, West RM, Dumesic JA, Pfleger BF. A process for microbial hydrocarbon synthesis: overproduction of fatty acids in Escherichia coli and catalytic conversion to alkanes. Biotechnol Bioeng 2010;106:193-202.

Li SJ, Cronan Jr JE. Growth rate regulation of Escherichia coli acetyl coenzyme A carboxylase, which catalyzes the first committed step of lipid biosynthesis. J Bacterio 1993;175:332-40.

Li Q, Du W, Liu D. Perspectives of microbial oils for biodiesel production. Appl Microbio Biotechnol 2008;80:749-56.

Liao JC, Hou SY, Chao YP. Pathway analysis, engineering and physiological considerations for redirecting central metabolism. Biotechnol Bioeng 1996;52:129-40.

Liu T, Vora H, Khosla C. Quantitative analysis and engineering of fatty acid biosynthesis in E. coli. Metab Eng 2010;12:378-86.
Liu X, Sheng J, Curtiss Iii R. Fatty acid production in genetically modified Cyanobacteria. Proc Natl Acad Sci 2011;108:6899-904

Liu H, Yu C, Feng D, Cheng T, Meng X, Liu W, et al. Production of extracellular fatty acid using engineered Escherichia coli. Microb Cell Fact 2012;11:41.

Lu X. A perspective: photosynthetic production of fatty acid-based biofuels in genetically engineered Cyanobacteria. Biotechnol Adv 2010;28:742-6.

Lu X, Vora H, Khosla C. Overproduction of free fatty acids in E. coli: implications for biodiesel production. Metab Eng 2008;10:333-9.

Lu W, Ness JE, Xie W, Zhang X, Minshull J, Gross RA. Biosynthesis of monomers for plastics from renewable oils. J Am Chem Soc 2010;132:15451-5.

Park JH, Lee SY. Towards systems metabolic engineering of microorganisms for amino acid production. Curr Opin Biotechnol 2008;19:454.

Park SJ, Ahn WS, Green PR, Lee SY. Production of poly (3-hydroxybutyrate-co3-hydroxyhexanoate) by metabolically engineered Escherichia coli strains. Biomacromolecules 2001;2:248-54

Picataggio S, Rohrer T, Deanda K, Lanning D, Reynolds R, Mielenz J, et al. Metabolic engineering of Candida tropicalis for the production of long-chain dicarboxylic acids. Nat Biotechnol 1992;10:894-8.

Ragauskas AJ, Williams CK, Davison BH, Britovsek G, Cairney J, Eckert CA, et al. The path forward for biofuels and biomaterials. Science 2006;311:484-9.

Schenk PM, Thomas-Hall SR, Stephens E, Marx UC, Mussgnug JH, Posten C, et al. Second generation biofuels: high-efficiency microalgae for biodiesel production. Bioenergy Res 2008:1:20-43.

Schirmer A, Rude MA, Li X, Popova E, del Cardayre SB. Microbial biosynthesis of alkanes. Science 2010;329:559-62

Schörken U, Kempers P. Lipid biotechnology: industrially relevant production processes. Eur J Lipid Sci Technol 2009;111:627-45

Sims REH, Mabee W, Saddler JN, Taylor M. An overview of second generation biofuel technologies. Bioresour Technol 2010;101:1570-80.

Steen EJ, Kang Y, Bokinsky G, Hu Z, Schirmer A, McClure A, et al. Microbial production of fatty-acid-derived fuels and chemicals from plant biomass. Nature 2010;463:559-62.

Stephanopoulos G. Challenges in engineering microbes for biofuels production. Science 2007:315:801-4

Voelker TA, Davies HM. Alteration of the specificity and regulation of fatty acid synthesis of Escherichia coli by expression of a plant medium-chain acyl-acyl carrier protein thioesterase. J Bacteriol 1994;176:7320-7.

Wang X, Li L, Zheng Y, Zou H, Cao Y, Liu H, et al. Biosynthesis of long chain hydroxyfatty acids from glucose by engineered Escherichia coli. Bioresour Technol 2012;114:561-6.

Wang W, Liu X, Lu X. Engineering Cyanobacteria to improve photosynthetic production of alka(e)nes. Biotechnol Biofuels 2013;6:69.

Wen F, Sun J, Zhao H. Yeast surface display of trifunctional minicellulosomes for simultaneous saccharification and fermentation of cellulose to ethanol. Appl Environ Microbiol 2010;76:1251-60.

You C, Chen H, Myung S, Sathitsuksanoh N, Ma H, Zhang X-Z, et al. Enzymatic transformation of nonfood biomass to starch. Proc Natl Acad Sci 2013;110:7182-7.

Zhang F, Carothers JM, Keasling JD. Design of a dynamic sensor-regulator system for production of chemicals and fuels derived from fatty acids. Nat Biotechnol 2012a;30: 354-9.

Zhang F, Ouellet M, Batth TS, Adams PD, Petzold CJ, Mukhopadhyay A, et al. Enhancing fatty acid production by the expression of the regulatory transcription factor FadR. Metab Eng 2012b;14:653-60.

Zhang J, Lu X, Li J-J. Conversion of fatty aldehydes into alk (a/e)nes by in vitro reconstituted cyanobacterial aldehyde-deformylating oxygenase with the cognate electron transfer system. Biotechnol Biofuels 2013:6:86.

Zheng Y-N, Li L-L, Liu Q, Yang J-M, Wang X-W, Liu W, et al. Optimization of fatty alcohol biosynthesis pathway for selectively enhanced production of C12/14 and C16/18 fatty alcohols in engineered Escherichia coli. Microb Cell Fact 2012;11:1-11. 